\title{
A Copper-based polycarbonyl coordination polymer as a cathode for
}

\section{Li-ion batteries}

Haoqi Su, ${ }^{\dagger}$ Yidan Song, ${ }^{\dagger}$ Yao Hu,,${ }^{\ddagger}$ Yanwei Ma,${ }^{\dagger}$ Wenlong Liu, ${ }^{\#}$ Hongjiang Liu $*{ }^{\ddagger}$ and

$$
\text { Qi Liu*` }
$$

†Jiangsu Key Laboratory of Advanced Catalytic Materials and Technology, and Jiangsu Province Key Laboratory of Fine Petrochemical Engineering, School of Petrochemical Engineering, Changzhou University, Changzhou, Jiangsu 213164, P. R.

\section{China}

Department of Chemistry, College of Science, Shanghai University, Shanghai

$$
\text { 200444, P. R. China }
$$

${ }^{\#}$ College of Chemistry and Chemical Engineering, Yangzhou University, Yangzhou,

$$
\text { Jiangsu 225002, P. R. China }
$$

\section{Scheme, Figures and Tables}<smiles></smiles>

PMDA<smiles>NCC(=O)O</smiles>

Gly<smiles>O=C(O)Cn1c(=O)c2cc3c(=O)n(CC(=O)O)c(=O)c3cc2c1=O</smiles>

BGPD

Scheme S1. Synthetic route of BGPD<smiles>O=C1OC(=O)c2ccc3c4c(ccc1c24)C(=O)OC3=O</smiles>

NTCDA

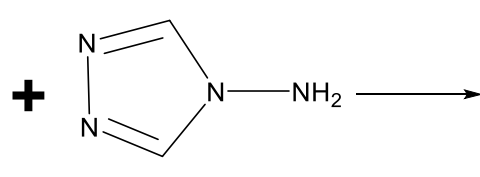

4-AT<smiles>O=C1c2ccc3c4c(ccc(c24)C(=O)N1n1cnnc1)C(=O)N(n1cnnc1)C3=O</smiles>

4-DTBPT

Scheme S2 Synthetic route of 4-DTBPT 
Table S1 Crystallographic data of $\mathrm{Cu}-\mathrm{CP}$

\begin{tabular}{|c|c|}
\hline Compound & $\mathrm{Cu}-\mathrm{CP}$ \\
\hline Empirical formula & $\mathrm{C}_{52} \mathrm{H}_{34} \mathrm{CuN}_{18} \mathrm{O}_{18}$ \\
\hline Formula weight & 1262.51 \\
\hline $\mathrm{T}(\mathrm{K})$ & 291(2) \\
\hline Cryst syst & triclinic \\
\hline space group & $P-1$ \\
\hline$a(\AA)$ & $9.0087(13)$ \\
\hline$b(\AA)$ & $10.2295(14)$ \\
\hline$c(\AA)$ & $15.198(2)$ \\
\hline$\alpha\left(^{\circ}\right)$ & $96.281(3)$ \\
\hline$\beta\left(^{\circ}\right)$ & $92.114(4)$ \\
\hline$\gamma\left({ }^{\circ}\right)$ & $105.962(3)$ \\
\hline$V\left(\AA^{3}\right)$ & $1335.2(3)$ \\
\hline$Z, D_{\text {calculated }}\left(\mathrm{g} \cdot \mathrm{cm}^{-3}\right)$ & $1,1.570$ \\
\hline $\operatorname{Mu}\left(\operatorname{Mo~K}_{\alpha}\right)\left(\mathrm{mm}^{-1}\right)$ & 0.505 \\
\hline$F(000)$ & 645 \\
\hline Crystal size $(\mathrm{mm})$ & $0.24 \times 0.16 \times 0.12$ \\
\hline$\theta$ range for data collection $\left(^{\circ}\right)$ & 2.34 to 25.83 \\
\hline Index ranges & $-10 / 11,-12 / 12,-18 / 12$ \\
\hline no. of total reflns & 8224 \\
\hline Unique reflns & $5199\left(R_{\mathrm{int}}=0.0312\right)$ \\
\hline Data / restraints / parameters & $5199 / 9 / 405$ \\
\hline Goodness- of-fit on $F^{2}$ & 1.015 \\
\hline$R_{1}, w R_{2}[I>2 \sigma(I)]$ & $0.0481,0.1182$ \\
\hline$R_{1}, w R_{2}$ (all data) & $0.0602,0.1182$ \\
\hline Largest diff. peak and hole $/ \mathrm{e} \cdot \AA^{-3}$ & 0.56 and -0.43 \\
\hline
\end{tabular}


Table S2. Main bond length $(\AA)$ and bond Angle $\left(^{\circ}\right)$ in $\mathrm{Cu}-\mathrm{CP}$

\begin{tabular}{|c|c|c|c|}
\hline $\mathrm{Cu}-\mathrm{CP}$ & & & \\
\hline$\overline{\mathrm{Cu} 1-\mathrm{N} 1^{\mathrm{iv}}}$ & $2.001(2)$ & $\mathrm{Cu} 1-\mathrm{N} 5^{\mathrm{iv}}$ & $2.011(2)$ \\
\hline $\mathrm{Cu} 1-\mathrm{N} 1$ & $2.001(2)$ & $\mathrm{Cu} 1-09^{\mathrm{iv}}$ & $2.408(2)$ \\
\hline $\mathrm{Cu}-\mathrm{N} 5$ & $2.011(2)$ & Cu1-O9 & $2.408(2)$ \\
\hline $\mathrm{N} 1^{\mathrm{iv}}-\mathrm{Cu} 1-\mathrm{N} 1$ & $180.00(11)$ & N5-Cu1-O9iv & $85.96(9)$ \\
\hline $\mathrm{N} 1^{\mathrm{iv}}-\mathrm{Cu} 1-\mathrm{N} 5$ & $90.17(9)$ & $\mathrm{N} 5^{\mathrm{iv}}-\mathrm{Cu} 1-\mathrm{O} 9^{\mathrm{iv}}$ & $94.04(9)$ \\
\hline N1-Cu1-N5 & $89.83(9)$ & $\mathrm{N} 1^{\mathrm{iv}}-\mathrm{Cu} 1-\mathrm{O} 9$ & $83.82(9)$ \\
\hline $\mathrm{N} 1^{\mathrm{iv}}-\mathrm{Cu} 1-\mathrm{N} 5^{\mathrm{iv}}$ & $89.83(9)$ & N1-Cu1-O9 & $96.18(9)$ \\
\hline $\mathrm{N} 1-\mathrm{Cu} 1-\mathrm{N} 5^{\mathrm{iv}}$ & $90.17(9)$ & N5-Cu1-O9 & $94.04(9)$ \\
\hline $\mathrm{N} 5-\mathrm{Cu} 1-\mathrm{N} 5^{\mathrm{iv}}$ & 180.0 & $\mathrm{~N} 5^{\mathrm{iv}}-\mathrm{Cu} 1-\mathrm{O} 9$ & $85.96(9)$ \\
\hline $\mathrm{N} 1^{\mathrm{iv}}-\mathrm{Cu} 1-\mathrm{O} 9^{\mathrm{iv}}$ & $96.18(9)$ & O9iv-Cu1-O9 & 180.0 \\
\hline $\mathrm{N} 1-\mathrm{Cu} 1-\mathrm{O} 9^{\mathrm{iv}}$ & $83.82(9)$ & & \\
\hline
\end{tabular}

Symmetry codes: (i) $-\mathrm{x}+1,-\mathrm{y},-\mathrm{z}$; (ii) $-\mathrm{x},-\mathrm{y},-\mathrm{z}$; (iii) $-\mathrm{x}-1,-\mathrm{y}+1,-\mathrm{z}$; (iv) $-\mathrm{x},-\mathrm{y}+1$, $-\mathrm{z}+1$.

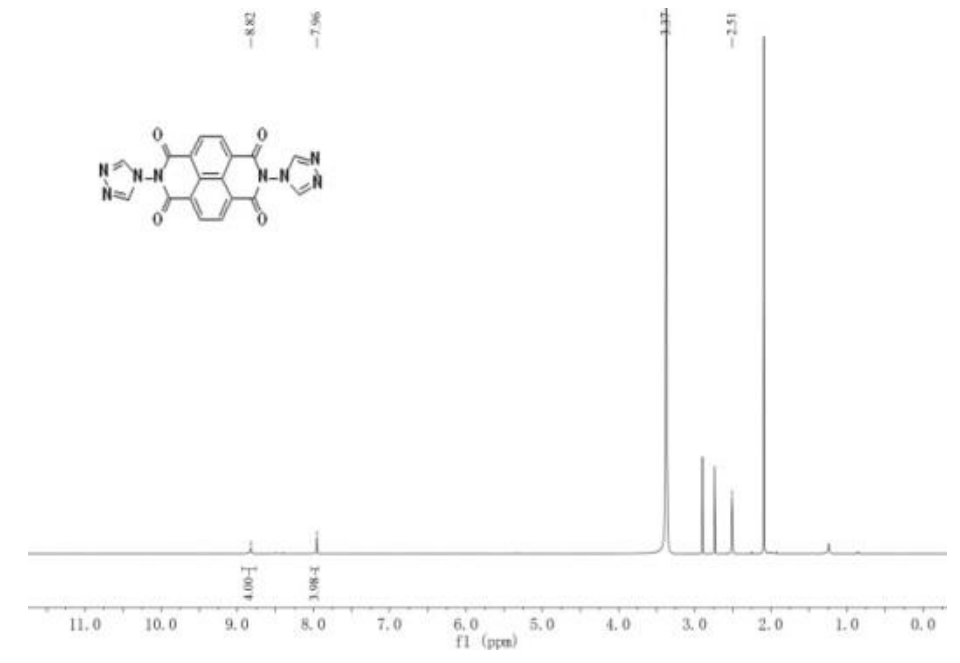

Figure S1. Liquid state ${ }^{1} \mathrm{H}$ NMR of 4-DTBPT.

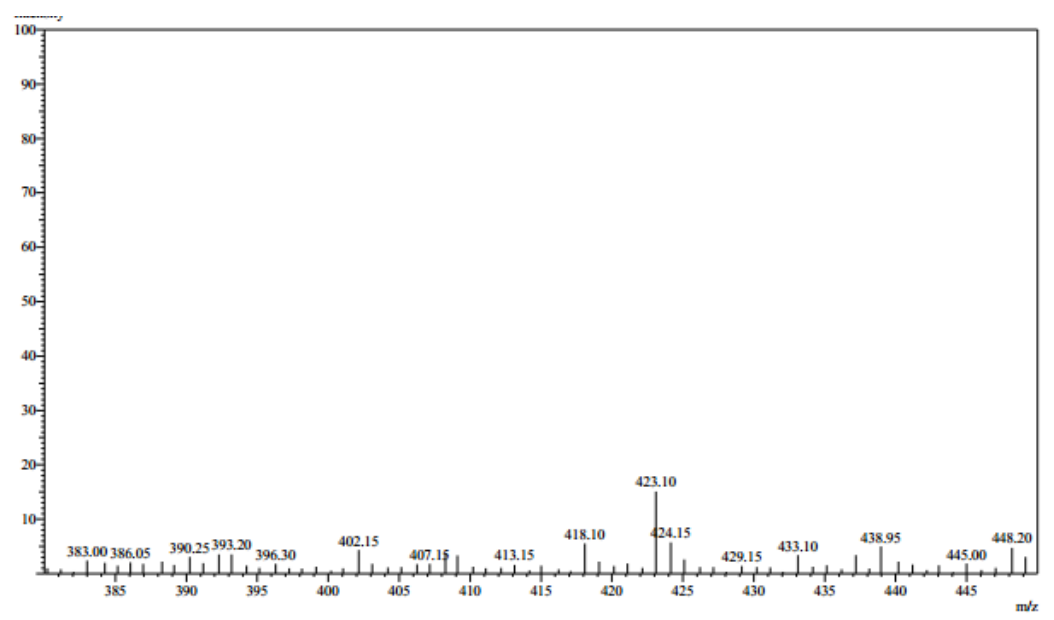

Figure S2. Mass spectra of 4-DTBPT 
Table S3. Hydrogen bond distances $(\AA)$ and angles $\left({ }^{\circ}\right)$ for $\mathrm{Cu}-\mathrm{CP}$

\begin{tabular}{lcccc}
\hline $\mathbf{D}-\mathbf{H} \cdots \mathbf{A}$ & $\mathbf{D}-\mathbf{H} / \AA$ & $\mathbf{H} \cdots \mathbf{A} / \AA$ & $\mathbf{D} \cdots \mathbf{A} / \AA$ & $\mathbf{D}-\mathbf{H} \cdots \mathbf{A} /{ }^{\circ}$ \\
\hline $\mathrm{O}(3)-\mathrm{H}(3 \mathrm{X}) \cdots \mathrm{O}(2)$ & 0.96 & 1.62 & $2.388(4)$ & 134 \\
$\mathrm{C}(1)-\mathrm{H}(1) \cdots \mathrm{O}(4)$ & 0.93 & 2.32 & $2.691(4)$ & 103 \\
$\mathrm{C}(1)-\mathrm{H}(1) \cdots \mathrm{O}(1)^{\mathrm{i}}$ & 0.93 & 2.36 & $2.734(4)$ & 104 \\
$\mathrm{C}(6)-\mathrm{H}(6) \cdots \mathrm{O}(4)$ & 0.93 & 2.44 & $3.161(4)$ & 135 \\
$\mathrm{C}(6)-\mathrm{H}(6) \cdots \mathrm{O}(8)^{\mathrm{ii}}$ & 0.93 & 2.55 & $3.124(4)$ & 120 \\
$\mathrm{C}(7)-\mathrm{H}(7) \cdots \mathrm{O}(9)^{\mathrm{iii}}$ & 0.93 & 2.47 & $2.984(4)$ & 115 \\
$\mathrm{C}(15)-\mathrm{H}(15) \cdots \mathrm{O}(3)^{\mathrm{iv}}$ & 0.93 & 2.58 & $3.393(4)$ & 147 \\
$\mathrm{C}(22)-\mathrm{H}(22) \cdots \mathrm{O}(7)^{\mathrm{v}}$ & 0.93 & 2.58 & $3.315(3)$ & 136 \\
$\mathrm{C}(26)-\mathrm{H}(26) \cdots \mathrm{N}(6)$ & 0.93 & 2.61 & $3.352(5)$ & 137
\end{tabular}

Symmetry code: ${ }^{\text {i }:} 1-x,-y,-z ;$ ii: $1+x, y, z ;{ }^{\text {iii: }}-x, 1-y, 1-z ;{ }^{\text {iv }}:-1+x, y, z ;{ }^{\mathrm{v}}:-x, 1-y,-z$;

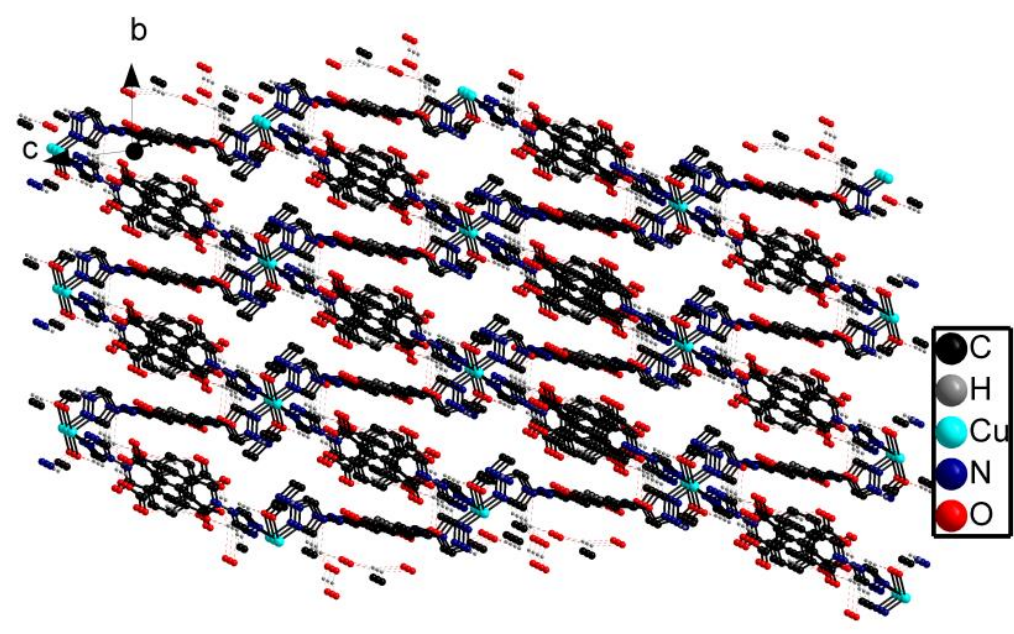

Figure S3. Three-dimensional structure of $\mathrm{Cu}-\mathrm{CP}$

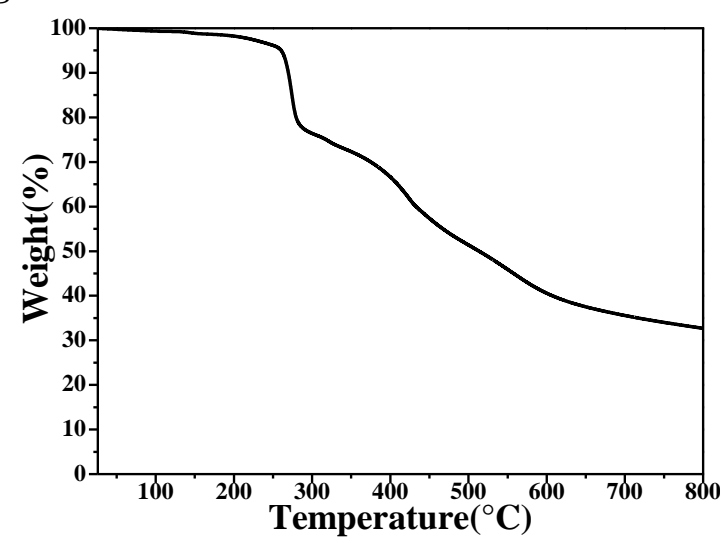

Figure S4. Thermogravimetric curve of $\mathrm{Cu}-\mathrm{CP}$. 


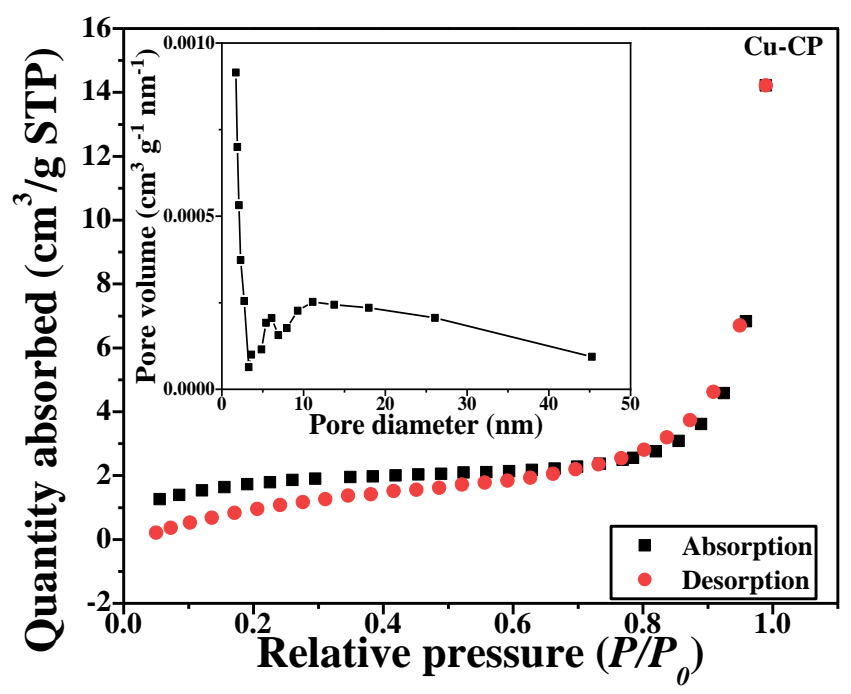

Figure S5. Nitrogen adsorption-desorption isotherm for the $\mathrm{Cu}-\mathrm{CP}$ sample and the inset is pore size distribution curve. 
Table S4. Specific capacitance of the $\mathrm{Cu}-\mathrm{CP}$ electrode in this study, compared with some $\mathrm{CP} / \mathrm{MOF}$-based electrodes reported in previous literature.

\begin{tabular}{|c|c|c|c|c|c|c|}
\hline $\mathrm{MOF} / \mathrm{CP}$ & $\begin{array}{l}\text { Content of } \\
\text { active } \\
\text { materials }\end{array}$ & $\begin{array}{l}\text { Specific } \\
\text { Capacity } \\
\left(\mathrm{mAh} \mathrm{g}^{-1}\right)\end{array}$ & $\begin{array}{l}\text { Current } \\
\text { Density } \\
\left(\mathrm{mA} \mathrm{g}^{-1}\right) / \\
\text { C rate }\end{array}$ & Cycle number & $\begin{array}{l}\text { Voltage } \\
(\mathrm{V} \text { vs. } \\
\left.\mathrm{Li} / \mathrm{Li}^{+}\right)\end{array}$ & Ref. \\
\hline MIL-53(Fe) & $85 \%$ & 70 & 50 & 50 cycles & $1.5-3.5$ & (S1) \\
\hline MIL-68 & $70 \%$ & 32 & $\mathrm{C} / 10$ & 12 cycles & $1.5-3.5$ & $(\mathrm{~S} 2)$ \\
\hline $\mathrm{Cu}(2,7-\mathrm{AQDC})$ & $10 \%$ & 105 & $1 \mathrm{~mA}$ & 50 cycles & $1.7-4.0$ & (S3) \\
\hline MIL-47 & $65 \%$ & 60 & 10 & 20 cycles & $1.8-3.2$ & $(\mathrm{~S} 4)$ \\
\hline MIL-132(K) & $70 \%$ & 40 & $10 \mathrm{C}$ & 5 cycles & $2.3-3.8$ & (S5) \\
\hline MIL-136(Ni) & $70 \%$ & 20 & $10 \mathrm{C}$ & 5 cycles & $2.0-4.3$ & (S6) \\
\hline MIL-136'(Ni) & $70 \%$ & 42 & $10 \mathrm{C}$ & 6 cycles & $2.3-4.5$ & (S6) \\
\hline Cu-TCA & $45 \%$ & 45 & $1 \mathrm{C}$ & 200 cycles & $1.8-3.4$ & (S7) \\
\hline MIL-101 & $70 \%$ & 22 & $\mathrm{C} / 20$ & 30 cycles & $2.0-3.5$ & (S8) \\
\hline MIL-101(Fe) & & 72 & $0.2 \mathrm{C}$ & 100 cycles & $2.0-4.2$ & (S9) \\
\hline $\begin{array}{l}\mathrm{Cd}(\mathrm{II}) \mathrm{MOFs} / \mathrm{Co}(\mathrm{II}) \\
\mathrm{MOFs}\end{array}$ & $45 \%$ & $37 / 3$ & 100 & 50 cycles & $1.8-3.4$ & (S10) \\
\hline Mn-MOF & $10 \%$ & 190 & $1 \mathrm{~mA}$ & 50 cycles & $1.3-4.5$ & (S11) \\
\hline NiDI & $70 \%$ & 50 & 250 & 300 cycles & $2.0-4.5$ & (S12) \\
\hline $\mathrm{CuPcOH}$ & $33.3 \%$ & 55 & 130 & 200 cycles & $2.0-4.4$ & (S13) \\
\hline$\left[\mathrm{Li}_{2}\left(\mathrm{C}_{6} \mathrm{H}_{2} \mathrm{O}_{4}\right)\right]$ & $60 \%$ & 137 & 100 & 10 cycles & $1.5-3.5$ & (S14) \\
\hline LiFe-MOF & $50 \%$ & 128 & 20 & 200 cycles & $1.5-4.5$ & (S15) \\
\hline DS-Cu-MOF & $30 \%$ & 119 & 50 & 50 cycles & $2.4-4.1$ & (S16) \\
\hline$\left[\mathrm{CuL}(\mathrm{DMF})_{2}\right]_{\mathrm{n}}$ & $30 \%$ & 120 & 80 & 30 cycle & $1.5-3.6$ & (S17) \\
\hline $\mathrm{Cu}_{3}(\mathrm{HHTP})_{2}$ & $80 \%$ & 85 & $1 \mathrm{C}$ & s100 cycles & $1.7-3.5$ & (S18) \\
\hline 1D-DS-Co-MOF & $30 \%$ & 35 & 200 & 100 cycles & $2.5-3.2$ & (S19) \\
\hline 2D-DS-Cu-MOF & $30 \%$ & 38 & 50 & 100 cycles & $2.4-4.1$ & (S19) \\
\hline 3D-DS-Mn-MOF & $30 \%$ & 48 & 200 & 100 cycles & $1.7-4.2$ & (S19) \\
\hline 1D-DS-Cu-CP & $30 \%$ & 50 & 50 & 2 cycles & $2.4-4.2$ & (S19) \\
\hline 3D-DS-Mn-CP & $30 \%$ & 25 & 200 & 2 cycles & $1.8-3.6$ & (S19) \\
\hline Fe-MOF & $80 \%$ & 147 & 40 & 50 cycles & $1.8-4.2$ & (S20) \\
\hline $\mathrm{Zn}-\mathrm{CP}$ & $10 \%$ & 57 & $1 \mathrm{C}$ & 1000 cycles & $1.4-4.0$ & (S21) \\
\hline Cu-THQ & $70 \%$ & 387 & 50 & 100 & $1.2-4.0$ & (S22) \\
\hline $\mathrm{Cu}-\mathrm{CP}$ & $30 \%$ & 40.3 & 50 & 50 cycles & $1.5-4.5$ & $\begin{array}{l}\text { This } \\
\text { work }\end{array}$ \\
\hline
\end{tabular}


Table S5. The fitting values of the impedance spectra at different cycles

\begin{tabular}{lllllllll}
\hline Sample & $R_{1}$ & $R_{\mathrm{S}}$ & CPE1-T & CPE1-P & $W 1-\mathrm{R}$ & $R_{\mathrm{ct}}$ & CPE2-T & CPE2-P \\
\hline After 1 cycle & 7.6 & 27.0 & $2.99 \times 10^{-5}$ & 0.73 & 10.83 & 9.39 & $5.6 \times 10^{-3}$ & 0.78 \\
After 50 cycles & 7.7 & 29.16 & $2.8 \times 10^{-6}$ & 0.74 & 8.35 & 10.05 & $6 \times 10^{-3}$ & 0.74 \\
\hline
\end{tabular}

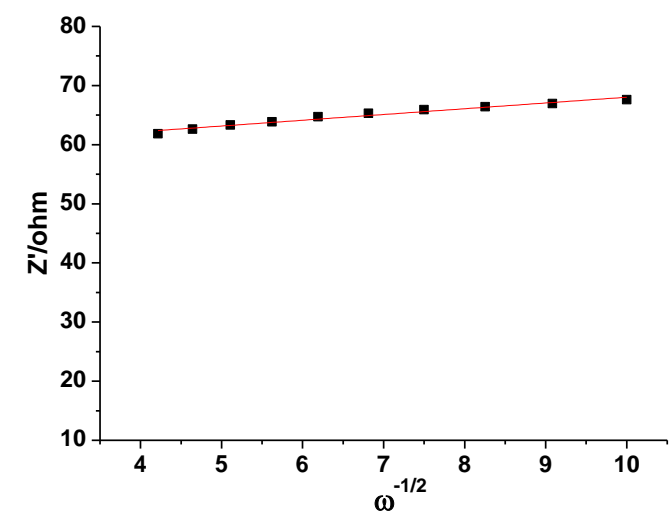

Figure S6. The linear fitting of the $Z^{\prime} v s$ square root of frequency $\left(\omega^{-1 / 2}\right)$ relationship, where $\omega$ is the angle frequency and $Z^{\prime}$ is the real part of Nyquist impedance of the $\mathrm{Cu}-\mathrm{CP}$ electrode after 1 cycle.

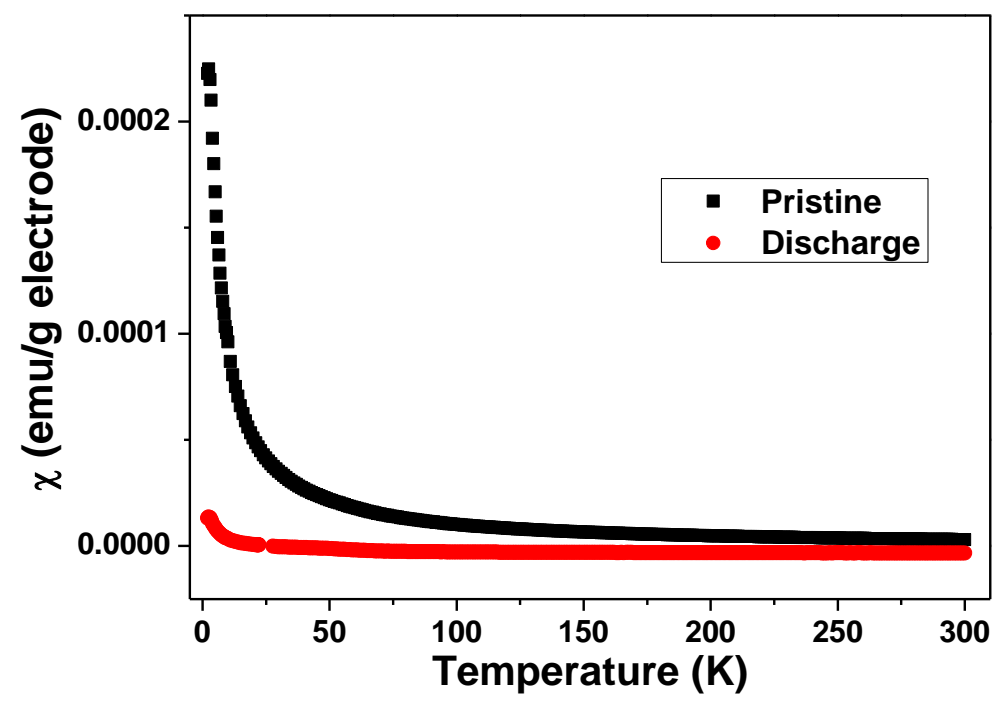

Figure S7. Magnetic susceptibilities $(\chi)$ for selected samples in the temperature range of 2-300K: pristine electrode prepared with $\mathrm{Cu}-\mathrm{CP}(\mathbf{a})$, after the first discharge to 1.5 V (०). 


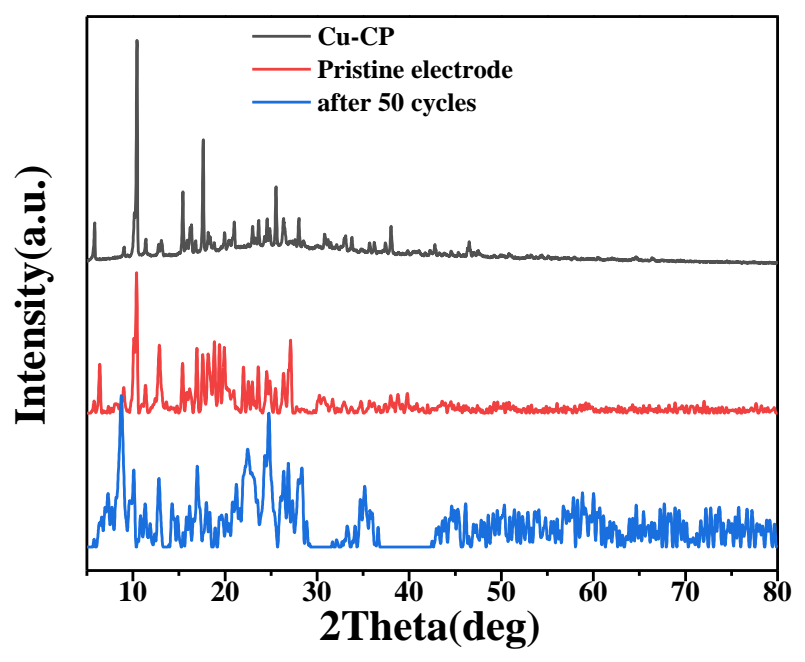

Figure S8. $\mathrm{XRD}$ patterns of $\mathrm{Cu}-\mathrm{CP}$, the $\mathrm{Cu}-\mathrm{CP}$ (1) prinstine electrode containing $\mathrm{Cu}-\mathrm{CP}$, polyvinylidene fluoride, and acetylene black (60 wt $\%: 30 \mathrm{wt} \%: 10 \mathrm{wt} \%$ ), and the prinstine electrode after 50 cycles.<smiles>O=C1c2ccc3c4c(ccc(c24)C(=O)N1n1cnnc1)C(=O)N(n1cnnc1)C3=O</smiles>
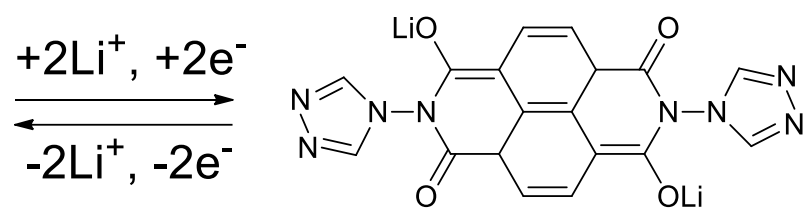

Figure S9. The possible mechanism of lithium ions intercalation/deintercalation for 4-DTBPT

\section{References}

S1. Férey, G.; Millange, F.; Morcrette, M.; Serre, C.; Doublet, M. L.; Grenèche, J. M.;

Tarascon, J. M. Mixed-valence Li/Fe-Based Metal-Organic Frameworks with Both

Reversible Redox and Sorption Properties. Angew. Chem. Int. Ed. 2007, 46, 3259-3263.

S2. Fateeva, A.; Horcajada, P.; Devic, T.; Serre, C.; Marrot, J.; Grenèche, J. M.; Morcrette, M.; Tarascon, J. M.; Maurin, G.; Férey, G. Synthesis, Structure, Characterization, and Redox Properties of the Porous MIL-68(Fe) Solid. Eur. J. Inorg. Chem. 2010, 2010, 3789-3794.

S3. Zhang, Z.; Yoshikawa, H.; Awaga, K. Monitoring the solid-state electrochemistry 
of $\mathrm{Cu}(2,7-\mathrm{AQDC})$ (AQDC = anthraquinone dicarboxylate) in a lithium battery: coexistence of metal and ligand redox activities in a metal-organic framework. J. Am. Chem. Soc. 2014, 136, 16112-16115.

S4. Kaveevivitchai, W.; Jacobson, A. J. Exploration of vanadium benzenedicarboxylate as a cathode for rechargeable lithium batteries. J. Power Sources 2015, 278, 265-273.

S5. Nguyen T. L. A.; Demir-Cakan, R.; Devic, T.; Morcrette, M.; Ahnfeldt, T.; Auban-Senzier, P.; Stock, N.; Goncalves, A. M.; Filinchuk, Y.; Tarascon, J. M.; Ferey, G. 3-D coordination polymers based on the tetrathiafulvalenetetracarboxylate (TTF-TC) derivative: synthesis, characterization, and oxidation issues. Inorg. Chem. 2010, 49, 7135-43.

S6. Nguyen T. L. A.; Demir-Cakan, R.; Devic, T.; Morcrette, M.; Ahnfeldt, T.; Auban-Senzier, P.; Stock, N.; Goncalves, A. M.; Filinchuk, Y.; Tarascon, J. M.; Férey, G. 3-D coordination polymers based on the tetrathiafulvalenetetracarboxylate (TTF-TC) derivative: synthesis, characterization, and oxidation issues. Inorg. Chem. 2010, 49, 7135-7143.

S7. Peng, Z.; Yi, X.; Liu, Z.; Shang, J.; Wang, D. Triphenylamine-Based Metal-Organic Frameworks as Cathode Materials in Lithium-Ion Batteries with Coexistence of Redox Active Sites, High Working Voltage, and High Rate Stability. ACS Appl. Mater. Interfaces 2016, 8, 14578-14585.

S8. Shin, J.; Kim, M.; Cirera, J.; Chen, S.; Halder, G. J.; Yersak, T. A.; Paesani, F.; Cohen, S. M.; Meng, Y. S. MIL-101(Fe) as a lithium-ion battery electrode material: a relaxation and intercalation mechanism during lithium insertion. J. Mater. Chem. A 2015, 3, 4738-4744.

S9. Yamada, T.; Shiraishi, K.; Kitagawa, H.; Kimizuka, N. Applicability of MIL-101(Fe) as a cathode of lithium ion batteries. Chem. Commun. 2017, 53, 
8215-8218.

S10. Tian, B.; Ning, G. H.; Gao, Q.; Tan, L. M.; Tang, W.; Chen, Z.; Su, C.; Loh, K.

P. Crystal Engineering of Naphthalenediimide-Based Metal-Organic Frameworks:

Structure-Dependent Lithium Storage. ACS Appl. Mater. Interfaces 2016, 8, 31067-31075.

S11. Zhang, Z.; Yoshikawa, H.; Awaga, K. Discovery of a "Bipolar Charging" Mechanism in the Solid-State Electrochemical Process of a Flexible Metal-Organic Framework. Chem. Mater. 2016, 28, 1298-1303.

S12. Wada, K.; Sakaushi, K.; Sasaki, S.; Nishihara, H. Multielectron-Transfer-based Rechargeable Energy Storage of Two-Dimensional Coordination Frameworks with Non-Innocent Ligands. Angew. Chem. Int. Ed. 2018, 57, 8886-8890.

S13. Nagatomi, H.; Yanai, N.; Yamada, T.; Shiraishi, K.; Kimizuka, N. Synthesis and Electric Properties of a Two-Dimensional Metal-Organic Framework Based on Phthalocyanine. Chem. Eur. J. 2018, 24, 1806-1810.

S14. Xiang, J.; Chang, C.; Li, M.; Wu, S.; Yuan, L.; Sun, J. A Novel Coordination Polymer as Positive Electrode Material for Lithium Ion Battery. Cryst. Growth Des. 2008, $8,280-282$.

S15. Schmidt, S.; Sheptyakov, D.; Jumas, J. C.; Medarde, M.; Benedek, P.; Novak, P.; Sallard, S.; Villevieille, C. Lithium Iron Methylenediphosphonate: A Model Material for New Organic-Inorganic Hybrid Positive Electrode Materials for Li Ion Batteries. Chem. Mater. 2015, 27, 7889-7895.

S16. Shimizu, T.; Wang, H.; Tanifuji, N.; Matsumura, D.; Yoshimura, M.; Nakanishi, K.; Ohta, T.; Yoshikawa, H. Rechargeable Batteries Based on Stable Redox Reactions of Disulfide Included in a Metal-Organic Framework as Ligands. Chem. Lett. 2018, 47, 678-681.

S17. Chang, C. H.; Li, A. C.; Popovs, I.; Kaveevivitchai, W.; Chen, J. L.; Chou, K. C.; Kuo, T. S.; Chen, T. H. Elucidating metal and ligand redox activities of a copper-benzoquinoid coordination polymer as the cathode for lithium-ion batteries. $J$. 
Mater. Chem. A, 2019, 7, 23770-23774.

S18. Gu, S.; Bai, Z.; Majumder, S.; Huang, B.; Chen, G. Conductive metal-organic framework with redox metal center as cathode for high rate performance lithium ion battery. J. Power Sources 2019, 429, 22-29.

S19. Shimizu, T.; Wang, H.; Matsumura, D.; Mitsuhara, K.; Ohta, T.; Yoshikawa, H. Porous Metal-Organic Frameworks Containing Reversible Disulfide Linkages as Cathode Materials for Lithium-Ion Batteries. ChemSusChem 2020, 13, 2256-2263.

S20. Ziebel, M. E.; Gaggioli, C. A.; Turkiewicz, A. B.; Ryu, W.; Gagliardi, L.; Long, J. R. Effects of Covalency on Anionic Redox Chemistry in Semiquinoid-Based Metal-Organic Frameworks. J. Am. Chem. Soc. 2020, 142, 2653-2664.

S21. Murata, T.; Koide, T.; Nobukuni, H.; Tsuji, R.; Morita, Y. 2D Coordination Network of Trioxotriangulene with Multiple Redox Abilities and Its Rechargeable Battery Performance. Int. J. Mol.Sci. 2020, 21, 4273.

S22. Jiang, Q.; Xiong, P.; Liu, J.; Xie, Z.; Wang, Q.; Yang, X. Q.; Hu, E.; Cao, Y.; Sun, J.; Xu, Y.; Chen, L. A Redox-Active 2D Metal-Organic Framework for Efficient Lithium Storage with Extraordinary High Capacity. Angew. Chem. Int. Ed. 2020, 59, 5273-5277. 